DFTT-20/2000

May 2000

\title{
Noncommutative geometry and physics: a review of selected recent results
}

\author{
Leonardo Castellani \\ Dipartimento di Scienze e Tecnologie Avanzate, East Piedmont University, Italy; \\ Dipartimento di Fisica Teorica and Istituto Nazionale di Fisica Nucleare \\ Via P. Giuria 1, 10125 Torino, Italy. \\ castellani@to.infn.it
}

\begin{abstract}
This review is based on two lectures given at the 2000 TMR school in Torino*. We discuss two main themes: i) Moyal-type deformations of gauge theories, as emerging from M-theory and open string theories, and ii) the noncommutative geometry of finite groups, with the explicit example of $Z_{2}$, and its application to Kaluza-Klein gauge theories on discrete internal spaces.
\end{abstract}

\footnotetext{
* TMR school on contemporary String Theory and Brane Physics

January 26 - February 2, 2000, Torino, Italy.
} 


\section{Introduction}

Noncommutativity of coordinates is not a surprising occurrence in physics, quantum phase space being the first example that comes to mind. In fact some early considerations on its "quantized" differential geometry can be found in [1]. This particular operator algebra has inspired the idea of spacetime coordinates as noncommuting operators. The idea has been explored since quite some time [2] in various directions, one main motivation being that the relation:

$$
\left[x^{\mu}, x^{\nu}\right]=i \theta^{\mu \nu}
$$

embodies an uncertainty principle that smears the spacetime picture at distances shorter than $\sqrt{\theta}$, and therefore a natural cutoff when using a quantum field theory to describe natural phenomena. Since "measuring" spacetime geometry under distances smaller than the Planck length $L_{P}$ is not accessible even to Gedanken experiments (at this scale the curvature radius of spacetime becomes of the order of a probe particle wavelength), relation (1.1) seems to make good physical sense when $\sqrt{\theta} \approx L_{P}$. Thus a quantum theory of gravity containing or predicting relation (1.1) would have a good chance to be intrinsically regulated.

String theories have been pointing towards a noncommuting scenario already in the 80's [3]. More recently Yang-Mills theories on noncommutative spaces have emerged in the context of $M$-theory compactified on a torus in the presence of constant background three-form field [4], or as low-energy limit of open strings in a backround $B$-field [5-11], describing the fluctuations of the $D$-brane world volume - As observed for example in [9], noncommutativity in open string theories is to be expected at some level, since open string vertex operators are inserted along a one-dimensional line, i.e. the boundary of the world sheet: the points of insertion are canonically ordered, so that the product of two such operators depends on their order of insertion. For a comprehensive account on noncommutativity in string theory and M-theory we refer to D. Bigatti's lectures [12], and to earlier reviews (for ex. [13).

The first part of this review concerns a short description of noncommutative Yang-Mills theories, with emphasis on the algebraic structure, that is on the (noncommutative) Moyal product, and with some remarks on the relations between deformed products and quantization rules. Recent results on perturbative aspects of noncommutative scalar field theories are recalled.

The second part is devoted to the differential geometry of finite groups. The general theory is illustrated in the case of $Z_{2}$. As a physical application, we construct a gauge theory on $M_{4} \times Z_{2}$, obtaining via a Kaluza-Klein mechanism a Higgs field (with the correct spontaneous symmetry-breaking potential and Yukawa couplings) in $d=4$ Minkowski spacetime $M_{4}$.

Noncommutative geometry (NCG) has a vast literature that we do not even attempt to cite. Reviews can be found in [14, 15, 16, 17]. We just mention some of its 
uses in physics not discussed in these two lectures: Connes' program of reconstructing the standard model from the NCG of suitable operator algebras 18; quantum groups, i.e. continuous deformations of Lie groups, and their NCG applied to gauge and gravity theories (see for ex. [19, 20, 21]), deformed quantum mechanics and solid state physics.

To find the geometry corresponding to a given algebraic structure is a fascinating and usually difficult task, whereas the inverse route is often much easier. A constructive starting point for NCG is to reformulate as much as possible the geometry of a manifold in terms of an algebra of functions defined on it ', and then to generalize the corresponding results of differential geometry to the case of a noncommutative algebra of functions. The main notion which is lost in this generalization is that of a point ("noncommutative geometry is pointless geometry").

\section{From sets of points to algebras of functions: $C^{*}$ algebras}

The primordial arena for geometry and topology are sets $V$ of points with some particular structure. Such a set we call "space". In many cases this set is completely characterized by an algebra of functions on it, so that all the information about $V$ can be retrieved from the functions alone.

Let us start with an elementary example: a finite dimensional vector space $V$. The functionals

$$
f: V \rightarrow \mathbb{R} \text { or } \mathbb{C}
$$

constitute the dual vector space $V^{*}$ isomorphic to $V$, a basis in $V^{*}$ being given by the functionals $x^{i}$, dual to the basis vectors $v_{j}$ of $V: x^{i}\left(v_{j}\right)=\delta_{j}^{i}$. The study of $V^{*}$ is completely equivalent to the study of $V$.

More generally consider a set $V$ of points, and the algebra of complex valued functions on $V, A=F u n(V)$. This algebra is clearly associative and commutative, with the usual pointwise product and sum: $(f \cdot g)(v)=f(v) g(v),(f+g)(v)=$ $f(v)+g(v),(\lambda f)(v)=\lambda f(v), \lambda \in \mathbb{C}$. The unit $I$ of the algebra is given by the function $I(v)=1, \forall v \in V$. As a simple example suppose again that $V$ has a finite number of elements. Then $A$ is of finite dimension as a vector space, and any $f \in A$ is expressible as

$$
f=f_{i} x^{i}, \quad x^{i}\left(v_{j}\right)=\delta_{j}^{i}
$$

where now $v_{j}$ are the elements of $V$. Note the multiplication rule:

$$
x^{i} x^{j}=\delta^{i j} x^{i}
$$

\footnotetext{
${ }^{1}$ For example tangent vectors on a manifold $V$ can be seen as derivations on the functions on $V$, etc.
} 
A norm can be defined in $A:\|f\| \equiv \max _{v \in V}|f(v)|$. Let $f^{*}$ be the complex conjugate of $f$, then

$$
\left\|f f^{*}\right\|=\|f\|^{2}
$$

A normed algebra with an involution $f \rightarrow f^{*}$ satisfying (2.4) is called a $C^{*}$ algebra. Thus $A=F u n(V)$ is a (commutative) $C^{*}$ algebra.

Conversely any $n$-dimensional commutative $C^{*}$ algebra can be considered as algebra of functions on a set of $n$ points. Note that commutativity is essential to interpret it as an algebra of functions on a set of points.

The finite dimensional example extends to infinite sets if they have a topology. In fact if $V$ is a compact space, then the algebra $C^{\circ}(V)$ of continuous functions on $V$ is a $C^{*}$ algebra. Conversely any $C^{*}$ algebra $A$ with a unit element is isomorphic to the algebra of continuous complex functions on some compact space $V$. This space is just the space of homomorphisms $\chi$ from $A$ to $C$ such that $\chi(I)=1$. The points of $V$ are then in 1-1 correspondence with irreducible representations of $A$. This is essentially the commutative Gel'fand-Naimark theorem.

Replacing now the commutative $A$ with a noncommutative $A$, the "space" may be hard to find: in most cases these algebras have non nontrivial homomorphisms into $\mathbb{C}$, so that the reconstruction of a space fails. But the existence of such a space may not be necessary, if one has transferred all the relevant information for a physical theory into the algebra $A$.

There are various ways to generalize to the noncommuting case. Continuous deformations of commutative $A$ into noncommutative $A$ include quantum groups (and quantum coset spaces) and deformations of Poisson structures, of which the noncommutative torus is a simple example. In these cases there is a set of continuous parameters that control the noncommutativity, and one recovers the commuting case (the "classical limit") for some specified values of these parameters.

On the other hand there are noncommutative algebras that are not connected to a commutative limit, as in the case of matrices with entries in $F u n(V)$. An example that we will work out in some detail in Section 4 is the differential geometry of finite groups: in this case $F u n(V)$ is commutative, but the differentials do not simply anticommute between themselves and do not commute with functions: hence a noncommutative differential geometry.

\section{Deformation quantization}

Consider the algebra of smooth functions on phase space. Deformation quantization essentially consists in deforming the usual commutative product between functions into an associative noncommutative product, the "star" product:

$$
A * B=A B+i \frac{\hbar}{2}\{A, B\}_{P B}+0\left(\hbar^{2}\right)
$$


where $\hbar$ is a parameter ( $\hbar \rightarrow 0$ corresponds to the commutative limit), and $\{A, B\}_{P B}$ is the Poisson bracket of the two phase space functions $A(q, p), B(q, p)$. Imposing associativity of the star product determines the higher $0\left(\hbar^{2}\right)$ terms up to equivalences that we discuss in next paragraph.

More generally, on a given manifold $X$ with a Poisson structure there is essentially one star product, modulo gauge equivalences that amount to linear redefinitions of the functions:

$$
A \rightarrow D(\hbar) A \equiv A+\hbar D_{1}(A)+\hbar^{2} D_{2}(A)+\ldots
$$

$D_{i}: F u n(X) \rightarrow F u n(X)$ being differential operators. This result was proved, in the sense of formal series expansions, in ref.s [22, 23]. That the linear automorphisms (3.2) are gauge transformations with respect to the star product can be understood as follows: consider the deformation of the ordinary product $A B$ due to $D(\hbar)$ :

$$
A * B=D(\hbar)^{-1}(D(\hbar)(A) D(\hbar)(B))
$$

This product is still commutative, and not essentially different from the ordinary one. Two $*$ products related by $D(\hbar)$ may therefore be considered equivalent. Thus deformation quantization yields a noncommutative algebra of functions for each Poisson structure on the manifold $X$. Poisson structures $\{$,$\} can be parametrized$ by an antisymmetric tensor $\theta^{i j}(x)$ such that $\{A, B\} \equiv \theta^{i j}(x)\left(\partial_{i} A\right)\left(\partial_{j} B\right)$, satisfying differential identities corresponding to the Jacobi identities of the Poisson bracket. The simplest Poisson structure is of course the Poisson bracket of ordinary (flat) phase-space, whose noncommutative algebra we consider in the following.

Historically the deformations (3.1) arose in studying the noncommutative structure of quantum mechanics, and this explains the word "quantization" and the appearance of the symbol $\hbar$ as deformation parameter. Consider for example the Weyl quantization rule $W$ (a linear map from the classical phase-space functions to the quantum operators) of the basic phase space monomial:

$$
q^{m} p^{n} \rightarrow W\left(q^{m} p^{n}\right)=\frac{1}{2^{n}} \sum_{k=0}^{n}\left(\begin{array}{c}
n \\
k
\end{array}\right) \hat{p}^{n-k} \hat{q}^{m} \hat{p}^{k}
$$

where $\hat{q}, \hat{p}$ are the quantum phase space operators. This rule amounts to sum on the permutations of all $\hat{p}$ and $\hat{q}$ considered as different objects, thus producing an hermitian operator. For example

$$
W\left(q p^{2}\right)=\frac{1}{4}\left(\hat{p}^{2} \hat{q}+2 \hat{p} \hat{q} \hat{p}+\hat{q} \hat{p}^{2}\right)
$$

Note that this rule can be efficiently restated as

$$
W\left(q^{m} p^{n}\right)=\left[\exp \left[-\frac{1}{2} i \hbar\left(\frac{\partial^{2}}{\partial q \partial p}\right)\right] q^{m} p^{n}\right]_{q \rightarrow \hat{q}, p \rightarrow \hat{p}}
$$


where the substitution $q \rightarrow \hat{q}, p \rightarrow \hat{p}$ occurs on each monomial $q^{r} p^{s}$ with $q$ 's ordered to the left. This formula may be checked to hold on the basic monomial, and extends therefore to any phase-space function $A(q, p)$ expressible as a power series:

$$
W(A(q, p))=: \exp \left[-\frac{1}{2} i \hbar\left(\frac{\partial^{2}}{\partial q \partial p}\right)\right] A(q, p):
$$

: $\quad$ indicating normal ordering ( $q$ preceding $p$ ) and substitution $q \rightarrow \hat{q}, p \rightarrow \hat{p}$. The map $W$ is invertible, i.e. there is a 1-1 correspondence between quantum operators and functions on phase-space. This is essentially the core of Moyal formalism 24, 25], enabling the study of quantum systems within the classical arena of phase-space via the inverse map $W^{-1}$.

Consider the product of two quantum operators $W(A), W(B)$ : the classical image $W^{-1}$ of their product is what is called the Moyal product $A * B$, and is given by Z $^{2}$

$$
W^{-1}(W(A) W(B)) \equiv A * B=A(q, p) \exp \left[i \frac{\hbar}{2} \triangle\right] B(q, p)
$$

where $\triangle$ is the bidifferential operator defining the Poisson bracket:

$$
A \triangle B \equiv\{A, B\}_{P B}
$$

i.e. $\triangle=\left(\frac{\overleftarrow{\partial}}{\partial q} \frac{\vec{\partial}}{\partial p}-\frac{\overleftarrow{\partial}}{\partial p} \frac{\vec{\partial}}{\partial q}\right)$. Clearly the Moyal product inherits the properties of the operator product, i.e. it is associative and noncommutative (unless the operators $W(A), W(B)$ happen to commute), and gives an explicit instance of the star product (3.1).

The Moyal bracket $\{A, B\}_{M}$ is given by the commutator:

$$
\{A, B\}_{M} \equiv A * B-B * A=2 i A \sin \left[\frac{\hbar}{2} \triangle\right] B
$$

and obviously has all the properties of a Lie bracket: it is bilinear, antisymmetric and satisfies Jacobi identities. The Moyal bracket is the image in classical phasespace of the commutator between quantum operators:

$$
\{A, B\}_{M}=W^{-1}([W(A), W(B)])
$$

cf. eq. (3.8).

Of course the Weyl map is not the only possible quantization rule. A classification of quantization rules and the construction of the corresponding noncommutative $*$ products and brackets can be found in [28]. In fact different quantization rules correspond to $*$ products connected by the gauge transformations (3.2).

\footnotetext{
${ }^{2}$ In fact the product was introduced by H. Groenewold [26] (and even earlier, less explicitly, by J. von Neumann [27]). We thank C. Zachos for bringing this to our attention.
} 
Similarly we can introduce noncommutativity in ordinary $\mathbb{R}^{d}$ spacetime via a new product on the $C^{*}$ algebra of $C^{\infty}$ complex functions:

$$
A * B(x) \equiv A(x) \exp \left[\frac{i}{2} \overleftarrow{\partial}_{\mu} \theta^{\mu \nu} \vec{\partial}_{\nu}\right] B(x)
$$

where $\theta^{\mu \nu}$ is constant, real and antisymmetric. Then the commutator of the coordinates $x^{\mu}$ computed with the star product yields precisely relation (1.1). By a change of coordinates $\theta$ can be reduced to the symplectic form:

$$
\theta=\left(\begin{array}{rrr}
0 & 1 & \\
-1 & 0 & \\
& & \ddots
\end{array}\right)
$$

Thus if $\theta$ has rank $r$ the relations (1.1) describe a spacetime with $\frac{r}{2}$ pairs of noncommuting coordinates (with the same algebraic structure as an $r$-dimensional phasespace) and $d-r$ coordinates that commute with all the others. In the $r$-dimensional subspace the star product coincides with the Moyal product discussed previously.

A noncommutative torus is obtained by considering periodic coordinates $0 \leq$ $x^{\mu}<2 \pi$. In the periodic case it is convenient to redefine the star product (3.12) as $A * B(x) \equiv A(x) \exp \left[\pi i \overleftarrow{\partial}_{\mu} \theta^{\mu \nu} \vec{\partial}_{\nu}\right] B(x)$ (which amounts to multiply $\theta$ by $2 \pi$ ), and to change variables:

$$
U_{\mu} \equiv e^{i x^{\mu}}
$$

The product between these new variables becomes:

$$
U_{\mu} * U_{\nu}=e^{\pi i \theta^{\mu \nu}} e^{i\left(x^{\mu}+x^{\nu}\right)}=e^{2 \pi i \theta^{\mu \nu}} U_{\nu} * U_{\mu}
$$

Notice that two noncommutative tori related by $\theta^{\mu \nu} \rightarrow \theta^{\mu \nu}+\Lambda^{\mu \nu}$, where $\Lambda^{\mu \nu}$ is antisymmetric with integer entries, are equivalent.

Quantum field theories on noncommutative spacetime (for a very partial list of ref.s see [29]-[38]) are then obtained by considering their ordinary action and replacing the usual product between fields with the $*$ product. Indeed the algebra of functions on noncommutative $R^{d}$ can be viewed as the algebra of ordinary functions on the usual $R^{d}$ with a deformed $*$ product. Thus we transfer the noncommutativity of spacetime to the noncommutativity of the product between functions, and then apply the usual perturbation theory. Because of the nonpolynomial character of the star product the resulting field theory is nonlocal. This kind of theories is under active study. We'll mention here only a few results.

Noncommutative scalar theories at the perturbative level have been investigated for example in [36]. The quadratic part of the action is the same as in the noncommutative theory, since $\int d^{d} x \phi * \phi=\int d^{d} x \phi \phi$ and likewise for the kinetic term (assuming suitable boundary conditions on $\phi$ that allow to drop total derivatives). Therefore propagators are the same as in the commutative case. The interactions 
however are modified: in momentum space an interaction vertex $\phi^{n}$ gives rise to an additional phase factor:

$$
V\left(k_{1}, k_{2}, \ldots, k_{n}\right)=e^{-\frac{i}{2} \sum_{i<j} k_{i} \times k_{j}}
$$

where $k_{i}$ is the momentum flowing into the vertex through the $i$ th $\phi$ and $k_{i} \times k_{j} \equiv$ $\left(k_{i}\right)_{\mu} \theta^{\mu \nu}\left(k_{j}\right)_{\nu}$. This is the only modification to the Feynman rules and its consequences have been investigated in [36], finding that $\theta$ dependence factorizes in planar graphs (i.e. the phase factor associated with the planar diagram does not contain internal momenta), yielding then essentially the same behaviour as in the noncommutative theory. Interesting differences arise in the non-planar diagrams: the one-loop diagrams turn out to be finite at generic values of the external momenta, a consequence of the rapid oscillations of phase factors of the type $e^{i p \times k}$ where $p$ is an external momentum and $k$ is the loop momentum. These factors disappear when $p_{\mu} \theta^{\mu \nu} \rightarrow 0$, and the nonplanar graphs become singular in this limit. This can be interpreted as a mixing between UV and IR divergences: turning on $\theta$ replaces the UV divergence with a $p \rightarrow 0$ IR divergence. Moreover, the commutative limit $\theta \rightarrow 0$ is not smooth 38 .

The quantum analysis is more complicated for noncommutative Yang-Mills theories. Classically the noncommutative $U(N)$ YM action is :

$$
S=\frac{1}{4 g^{2}} \int \operatorname{Tr}\left(F_{\mu \nu} * F_{\mu \nu}\right)
$$

where

$$
\begin{aligned}
& F_{\mu \nu}=\partial_{\mu} A_{\nu}-\partial_{\nu} A_{\mu}-i\left(A_{\mu} * A_{\nu}-A_{\nu} * A_{\mu}\right) \\
& A=A^{a} t^{a}, \quad \operatorname{Tr}\left(t^{a} t^{b}\right)=\delta^{a b}
\end{aligned}
$$

As already noticed in 39, the noncommutative gauge transformations:

$$
\begin{aligned}
& \delta_{\varepsilon} A_{\mu}=\partial_{\mu} \varepsilon-i\left(A_{\mu} * \varepsilon-\varepsilon * A_{\mu}\right) \\
& \delta_{\varepsilon} F_{\mu \nu}=-i\left(F_{\mu \nu} * \varepsilon-\varepsilon * F_{\mu \nu}\right)
\end{aligned}
$$

leave the action invariant. The perturbative quantum theory is the object of current research.

The extension of the AdS/CFT correspondence to backgrouds with constant $B$ field can shed some light on the nonperturbative regime of noncommutative field theories, see for ex. ref. [40].

Deformation quantization has been applied to numerous other physical systems, besides scalar and gauge theories and their supersymmetric versions [41, 42]. We mention for example gravity [43] and the bosonic string action [44]. 


\section{Dynamics on finite groups from their noncom- mutative geometry}

In this Section we present a systematic method for constructing field theories on finite groups. This method is based on the (noncommutative) differential geometry of finite groups, studied in ref.s [45, 46, 48, 47]. The general theory is applied to the simplest possible finite group, i.e. $Z_{2}$. The example of the simplest nonabelian finite group $S_{3}$ can be found in [47, 48, and in 47] a gravity-like theory on $S_{3}$ is discussed. Here we will use the NCG on $Z_{2}$ to formulate a $U(N)$ gauge theory coupled to Dirac fermions on $M_{4} \times Z_{2}$, yielding in $M_{4}$ (Minkowski spacetime) a Yang-Mills theory coupled to Dirac matter plus a Higgs sector with symmetry-breaking potential and Yukawa couplings to the fermions.

Differential calculi can be constructed on spaces that are more general than differentiable manifolds. Indeed the general algebraic construction of differential calculus in terms of Hopf structures [51] allows to extend the usual differential geometric quantities (connection, curvature, metric, vielbein etc.) to a variety of interesting spaces that include quantum groups, noncommutative spacetimes (i.e. quantum cosets), and discrete spaces.

In this lecture we concentrate on the differential geometry of finite group "manifolds". As discussed in [45, 47, 48], these spaces can be visualized as collections of points, corresponding to the finite group elements, and connected by oriented links according to the particular differential calculus we build on them. Although functions $f \in \operatorname{Fun}(G)$ on finite groups $G$ commute, the calculi that are constructed on $\operatorname{Fun}(G)$ by algebraic means are in general noncommutative, in the sense that differentials do not commute with functions, and the exterior product does not coincide with the usual antisymmetrization of the tensor product.

Among the physical motivations for finding differential calculi on finite groups we mention the possibility of using finite group spaces as internal spaces for KaluzaKlein compactifications of Yang-Mills, supergravity or superstring theories ( for example Connes' reconstruction of the standard model in terms of noncommutative geometry [18] can be recovered as Kaluza-Klein compactification of Yang-Mills theory on an appropriate discrete internal space). Differential calculi on discrete spaces can be of use in the study of integrable models, see for ex. ref. [49]. Finally gauge and gravity theories on finite group spaces may be used as lattice approximations. For example the action for pure Yang-Mills $\int F \wedge^{*} F$ considered on the finite group space $Z^{N} \times Z^{N} \times Z^{N} \times Z^{N}$, yields the usual Wilson action of lattice gauge theories, and $N \rightarrow \infty$ gives the continuum limit [50]. New lattice theories can be found by choosing different finite groups.

While the construction of the differential calculus on finite groups in ref.s 445, 46, 47, 48 uses the Hopf algebraic approach of Woronowicz [51, here this calculus will be presented without recourse to Hopf algebra techniques. Most of the content 
of next Section can be found in [47, 48], and is included here for self-consistency.

\subsection{Differential calculus on finite groups}

Let $G$ be a finite group of order $n$ with generic element $g$ and unit $e$. Consider Fun $(G)$, the set of complex functions on $G$. An element $f$ of $F u n(G)$ is specified by its values $f_{g} \equiv f(g)$ on the group elements $g$, and can be written as

$$
f=\sum_{g \in G} f_{g} x^{g}, \quad f_{g} \in \boldsymbol{C}
$$

where the functions $x^{g}$ are defined by

$$
x^{g}\left(g^{\prime}\right)=\delta_{g^{\prime}}^{g}
$$

Thus $\operatorname{Fun}(G)$ is a n-dimensional vector space, and the $n$ functions $x^{g}$ provide a basis. $\operatorname{Fun}(G)$ is also a commutative algebra, with the usual pointwise sum and product, and unit $I$ defined by $I(g)=1, \forall g \in G$. In particular:

$$
x^{g} x^{g^{\prime}}=\delta_{g, g^{\prime}} x^{g}, \quad \sum_{g \in G} x^{g}=I
$$

Consider now the left multiplication by $g_{1}$ :

$$
L_{g_{1}} g_{2}=g_{1} g_{2}, \quad \forall g_{1}, g_{2} \in G
$$

This induces the left action (pullback) $\mathcal{L}_{g_{1}}$ on Fun $(G)$ :

$$
\left.\mathcal{L}_{g_{1}} f\left(g_{2}\right) \equiv f\left(g_{1} g_{2}\right)\right|_{g_{2}}, \quad \mathcal{L}_{g_{1}}: \operatorname{Fun}(G) \rightarrow \text { Fun }(G)
$$

where $\left.f\left(g_{1} g_{2}\right)\right|_{g_{2}}$ means $f\left(g_{1} g_{2}\right)$ seen as a function of $g_{2}$. Similarly we can define the right action on $\operatorname{Fun}(G)$ as:

$$
\left(\mathcal{R}_{g_{1}} f\right)\left(g_{2}\right)=\left.f\left(g_{2} g_{1}\right)\right|_{g_{2}}
$$

For the basis functions we find easily:

$$
\mathcal{L}_{g_{1}} x^{g}=x^{g_{1}^{-1} g}, \quad \mathcal{R}_{g_{1}} x^{g}=x^{g g_{1}^{-1}}
$$

Moreover:

$$
\begin{aligned}
& \mathcal{L}_{g_{1}} \mathcal{L}_{g_{2}}=\mathcal{L}_{g_{1} g_{2}}, \quad \mathcal{R}_{g_{1}} \mathcal{R}_{g_{2}}=\mathcal{R}_{g_{2} g_{1}} \\
& \mathcal{L}_{g_{1}} \mathcal{R}_{g_{2}}=\mathcal{R}_{g_{2}} \mathcal{L}_{g_{1}}
\end{aligned}
$$

\section{Differential calculus}

A first-order differential calculus on $A$ is defined by 
i) a linear map $d: A \rightarrow \Gamma$, satisfying the Leibniz rule

$$
d(a b)=(d a) b+a(d b), \quad \forall a, b \in A ;
$$

The "space of 1-forms" $\Gamma$ is an appropriate bimodule on $A$, which essentially means that its elements can be multiplied on the left and on the right by elements of $A$ [more precisely $A$ is a left module if $\forall a, b \in A, \forall \rho, \rho^{\prime} \in \Gamma$ we have: $a\left(\rho+\rho^{\prime}\right)=$ $a \rho+a \rho^{\prime},(a+b) \rho=a \rho+b \rho, a(b \rho)=(a b) \rho, I \rho=\rho$. Similarly one defines a right module. A left and right module is a bimodule if $a(\rho b)=(a \rho) b]$. From the Leibniz rule $d a=d(I a)=(d I) a+I d a$ we deduce $d I=0$.

ii) the possibility of expressing any $\rho \in \Gamma$ as

$$
\rho=\sum_{k} a_{k} d b_{k}
$$

for some $a_{k}, b_{k}$ belonging to $A$.

To build a first order differential calculus on $F u n(G)$ we need to extend the algebra $A=\operatorname{Fun}(G)$ to a differential algebra of elements $x^{g}, d x^{g}$ (it is sufficient to consider the basis elements and their differentials). Note however that the $d x^{g}$ are not linearly independent. In fact from $0=d I=d\left(\sum_{g \in G} x^{g}\right)=\sum_{g \in G} d x^{g}$ we see that only $n-1$ differentials are independent. Every element $\rho=a d b$ of $\Gamma$ can be expressed as a linear combination (with complex coefficients) of terms of the type $x^{g} d x^{g^{\prime}}$. Moreover $\rho b \in \Gamma$ (i.e. $\Gamma$ is also a right module) since the Leibniz rule and the multiplication rule (4.3) yield the commutations:

$$
d x^{g} x^{g^{\prime}}=-x^{g} d x^{g^{\prime}}+\delta_{g^{\prime}}^{g} d x^{g}
$$

allowing to reorder functions to the left of differentials.

\section{Partial derivatives}

Consider the differential of a function $f \in F u n(g)$ :

$$
d f=\sum_{g \in G} f_{g} d x^{g}=\sum_{g \neq e} f_{g} d x^{g}+f_{e} d x^{e}=\sum_{g \neq e}\left(f_{g}-f_{e}\right) d x^{g} \equiv \sum_{g \neq e} \partial_{g} f d x^{g}
$$

We have used $d x^{e}=-\sum_{g \neq e} d x^{g}$ (from $\sum_{g \in G} d x^{g}=0$ ). The partial derivatives of $f$ have been defined in analogy with the usual differential calculus, and are given by

$$
\partial_{g} f=f_{g}-f_{e}=f(g)-f(e)
$$

Not unexpectedly, they take here the form of finite differences (discrete partial derivatives at the origin $e$ ). 


\section{Left and right covariance}

A differential calculus is left or right covariant if the left or right action of $G\left(\mathcal{L}_{g}\right.$ or $\mathcal{R}_{g}$ ) commutes with the exterior derivative $d$. Requiring left and right covariance in fact defines the action of $\mathcal{L}_{g}$ and $\mathcal{R}_{g}$ on differentials: $\mathcal{L}_{g} d b \equiv d\left(\mathcal{L}_{g} b\right), \forall b \in F u n(G)$ and similarly for $\mathcal{R}_{g} d b$. More generally, on elements of $\Gamma$ (one-forms) we define $\mathcal{L}_{g}$ as:

$$
\mathcal{L}_{g}(a d b) \equiv\left(\mathcal{L}_{g} a\right) \mathcal{L}_{g} d b=\left(\mathcal{L}_{g} a\right) d\left(\mathcal{L}_{g} b\right)
$$

and similar for $\mathcal{R}_{g}$. Computing for example the left and right action on the differentials $d x^{g}$ yields:

$$
\begin{aligned}
& \mathcal{L}_{g}\left(d x^{g_{1}}\right) \equiv d\left(\mathcal{L}_{g} x^{g_{1}}\right)=d x^{g^{-1} g_{1}}, \\
& \mathcal{R}_{g}\left(d x^{g_{1}}\right) \equiv d\left(\mathcal{R}_{g} x^{g_{1}}\right)=d x^{g_{1} g^{-1}}
\end{aligned}
$$

A differential calculus is called bicovariant if it is both left and right covariant.

\section{Left invariant one forms}

As in usual Lie group manifolds, we can introduce a basis in $\Gamma$ of left-invariant one-forms $\theta^{g}$ :

$$
\theta^{g} \equiv \sum_{h \in G} x^{h g} d x^{h} \quad\left(=\sum_{h \in G} x^{h} d x^{h g^{-1}}\right)
$$

It is immediate to check that indeed $\mathcal{L}_{k} \theta^{g}=\theta^{g}$. The relations (4.18) can be inverted:

$$
d x^{h}=\sum_{g \in G}\left(x^{h g}-x^{h}\right) \theta^{g}
$$

From $0=d I=d \sum_{g \in G} x^{g}=\sum_{g \in G} d x^{g}=0$ one finds:

$$
\sum_{g \in G} \theta^{g}=\sum_{g, h \in G} x^{h} d x^{h g^{-1}}=\sum_{h \in G} x^{h} \sum_{g \in G} d x^{h g^{-1}}=0
$$

Therefore we can take as basis of the cotangent space $\Gamma$ the $n-1$ linearly independent left-invariant one-forms $\theta^{g}$ with $g \neq e$ (but smaller sets of $\theta^{g}$ can be consistently chosen as basis, see later).

The $x, \theta$ commutations (bimodule relations) are easily derived:

$$
x^{h} d x^{g}=x^{h} \theta^{g^{-1} h}=\theta^{g^{-1} h} x^{g} \quad(h \neq g) \Rightarrow x^{h} \theta^{g}=\theta^{g} x^{h g^{-1}} \quad(g \neq e)
$$

implying the general commutation rule between functions and left-invariant oneforms:

$$
f \theta^{g}=\theta^{g} \mathcal{R}_{g} f
$$

Thus functions do commute between themselves (i.e. $\operatorname{Fun}(G)$ is a commutative algebra) but do not commute with the basis of one-forms $\theta^{g}$. In this sense the differential geometry of $\operatorname{Fun}(G)$ is noncommutative. 
The right action of $G$ on the elements $\theta^{g}$ is given by:

$$
\mathcal{R}_{h} \theta^{g}=\theta^{a d(h) g}, \quad \forall h \in G
$$

where $a d$ is the adjoint action of $G$ on itself, i.e. $a d(h) g \equiv h g h^{-1}$. Then bicovariant calculi are in 1-1 correspondence with unions of conjugacy classes (different from $\{e\})$ 45: if $\theta^{g}$ is set to zero, one must set to zero all the $\theta^{\operatorname{ad}(h) g}, \forall h \in G$ corresponding to the whole conjugation class of $g$.

We denote by $G^{\prime}$ the subset corresponding to the union of conjugacy classes that characterizes the bicovariant calculus on $G\left(G^{\prime}=\left\{g \in G \mid \theta^{g} \neq 0\right\}\right)$. Unless otherwise indicated, repeated indices are summed on $G^{\prime}$ in the following.

A bi-invariant (i.e. left and right invariant) one-form $\Theta$ is obtained by summing on all $\theta^{g}$ with $g \neq e$ :

$$
\Theta=\sum_{g \neq e} \theta^{g}
$$

\section{Exterior product}

For a bicovariant differential calculus on a Hopf algebra $A$ an exterior product, compatible with the left and right actions of $G$, can be defined by

$$
\theta^{g_{1}} \wedge \theta^{g_{2}}=\theta^{g_{1}} \otimes \theta^{g_{2}}-\theta^{g_{1}^{-1} g_{2} g_{1}} \otimes \theta^{g_{1}} \equiv \theta^{g_{1}} \otimes \theta^{g_{2}}-\Lambda_{g_{3} g_{4}}^{g_{1} g_{2}} \theta^{g_{3}} \otimes \theta^{g_{4}}
$$

where the tensor product between elements $\rho, \rho^{\prime} \in \Gamma$ is defined to have the properties $\rho a \otimes \rho^{\prime}=\rho \otimes a \rho^{\prime}, a\left(\rho \otimes \rho^{\prime}\right)=(a \rho) \otimes \rho^{\prime}$ and $\left(\rho \otimes \rho^{\prime}\right) a=\rho \otimes\left(\rho^{\prime} a\right)$.

Note that:

$$
\theta^{g} \wedge \theta^{g}=0 \quad(\text { no sum on } g)
$$

Left and right actions on $\Gamma \otimes \Gamma$ are simply defined by:

$$
\begin{aligned}
& \mathcal{L}_{h}\left(\rho \otimes \rho^{\prime}\right)=\mathcal{L}_{h} \rho \otimes \mathcal{L}_{h} \rho^{\prime} \\
& \mathcal{R}_{h}\left(\rho \otimes \rho^{\prime}\right)=\mathcal{R}_{h} \rho \otimes \mathcal{R}_{h} \rho^{\prime}
\end{aligned}
$$

(with the obvious generalization to $\Gamma \otimes \ldots \otimes \Gamma$ ) so that for example:

$$
\begin{aligned}
& \mathcal{L}_{h}\left(\theta^{i} \otimes \theta^{j}\right)=\theta^{i} \otimes \theta^{j}, \\
& \mathcal{R}_{h}\left(\theta^{i} \otimes \theta^{j}\right)=\theta^{a d(h) i} \otimes \theta^{a d(h) j}
\end{aligned}
$$

Compatibility of the exterior product with $\mathcal{L}$ and $\mathcal{R}$ means that

$$
\mathcal{L}\left(\theta^{i} \wedge \theta^{j}\right)=\mathcal{L} \theta^{i} \wedge \mathcal{L} \theta^{j}, \quad \mathcal{R}\left(\theta^{i} \wedge \theta^{j}\right)=\mathcal{R} \theta^{i} \wedge \mathcal{R} \theta^{j}
$$

only the second relation being nontrivial, and verifiable using the definition (4.25).

We can generalize the definition (4.25) to exterior products of $n$ one-forms:

$$
\theta^{i_{1}} \wedge \ldots \wedge \theta^{i_{n}} \equiv W_{j_{1} . . j_{n}}^{i_{1} . i_{n}} \theta^{j_{1}} \otimes \ldots \otimes \theta^{j_{n}}
$$


or in short-hand notation:

$$
\theta^{1} \wedge \ldots \wedge \theta^{n}=W_{1 . . n} \theta^{1} \otimes \ldots \otimes \theta^{n}
$$

where the labels $1 . . n$ in $W$ refer to index couples. The numerical coefficients $W_{1 \ldots n}$ are given through the recursion relation

$$
W_{1 \ldots n}=\mathcal{I}_{1 \ldots n} W_{1 \ldots n-1}
$$

where

$$
\mathcal{I}_{1 \ldots n}=1-\Lambda_{n-1, n}+\Lambda_{n-2, n-1} \Lambda_{n-1, n} \ldots-(-1)^{n} \Lambda_{12} \Lambda_{23} \cdots \Lambda_{n-1, n}
$$

$\Lambda$ being defined in (4.25) and $W_{1}=1$. The space of $n$-forms $\Gamma^{\wedge n}$ is therefore defined as in the usual case but with the new permutation operator $\Lambda$, and can be shown to be a bicovariant bimodule (see for ex. [52]), with left and right action defined as for $\Gamma \otimes \ldots \otimes \Gamma$ with the tensor product replaced by the wedge product.

\section{Exterior derivative}

Having the exterior product we can define the exterior derivative

$$
\begin{gathered}
d: \Gamma \rightarrow \Gamma \wedge \Gamma \\
d\left(a_{k} d b_{k}\right)=d a_{k} \wedge d b_{k},
\end{gathered}
$$

which can easily be extended to $\Gamma^{\wedge n}\left(d: \Gamma^{\wedge n} \rightarrow \Gamma^{\wedge(n+1)}\right)$, and has the following properties:

$$
\begin{gathered}
d\left(\rho \wedge \rho^{\prime}\right)=d \rho \wedge \rho^{\prime}+(-1)^{k} \rho \wedge d \rho^{\prime} \\
d(d \rho)=0 \\
\mathcal{L}_{g}(d \rho)=d \mathcal{L}_{g} \rho \\
\mathcal{R}_{g}(d \rho)=d \mathcal{R}_{g} \rho
\end{gathered}
$$

where $\rho \in \Gamma^{\wedge k}, \rho^{\prime} \in \Gamma^{\wedge n}, \Gamma^{\wedge 0} \equiv F u n(G)$. The last two properties express the fact that $d$ commutes with the left and right action of $G$.

\section{Tangent vectors}

Using (4.19) to expand $d f$ on the basis of the left-invariant one-forms $\theta^{g}$ defines the (left-invariant) tangent vectors $t_{g}$ :

$$
\begin{aligned}
d f & =\sum_{g \in G} f_{g} d x^{g}=\sum_{h \in G^{\prime}}\left(\mathcal{R}_{h^{-1}} f-f\right) \theta^{h} \equiv \\
& \equiv \sum_{h \in G^{\prime}}\left(t_{h} f\right) \theta^{h}
\end{aligned}
$$


so that the "flat" partial derivatives $t_{h} f$ are given by

$$
t_{h} f=\mathcal{R}_{h^{-1}} f-f
$$

The Leibniz rule for the flat partial derivatives $t_{g}$ reads:

$$
t_{g}\left(f f^{\prime}\right)=\left(t_{g} f\right) \mathcal{R}_{g^{-1}} f^{\prime}+f t_{g} f^{\prime}
$$

In analogy with ordinary differential calculus, the operators $t_{g}$ appearing in (4.42) are called (left-invariant) tangent vectors, and in our case are given by

$$
t_{g}=\mathcal{R}_{g^{-1}}-i d
$$

They satisfy the composition rule:

$$
t_{g} t_{g^{\prime}}=\sum_{h} C_{g, g^{\prime}}^{h} t_{h}
$$

where the structure constants are:

$$
C_{g, g^{\prime}}^{h}=\delta_{g^{\prime} g}^{h}-\delta_{g}^{h}-\delta_{g^{\prime}}^{h}
$$

and are $a d(G)$ invariant:

$$
C^{a d(h) g_{1}}{ }_{a d(h) g_{2}, a d(h) g_{3}}=C_{g_{2}, g_{3}}^{g_{1}}
$$

Note 4.1 : The exterior derivative on any $f \in F u n(G)$ can be expressed as a commutator of $f$ with the bi-invariant one-form $\Theta$ :

$$
d f=[\Theta, f]
$$

as one proves by using (4.22) and (4.42).

Note 4.2 : From the fusion rules (4.46) we deduce the "deformed Lie algebra" (cf. ref.s [51, 19, 52]):

$$
t_{g_{1}} t_{g_{2}}-\Lambda_{g_{1}, g_{2}}^{g_{3}, g_{4}} t_{g_{3}} t_{g_{4}}=C_{g_{1}, g_{2}}^{h} t_{h}
$$

where the $\boldsymbol{C}$ structure constants are given by:

$$
C_{g_{1}, g_{2}}^{g} \equiv C_{g_{1}, g_{2}}^{g}-\Lambda_{g_{1}, g_{2}}^{g_{3}, g_{4}} C_{g_{3}, g_{4}}^{g}=C_{g_{1}, g_{2}}^{g}-C_{g_{2}, g_{2} g_{1} g_{2}^{-1}}^{g}=\delta_{g_{1}}^{a d\left(g_{2}^{-1}\right) g}-\delta_{g_{1}}^{g}
$$

and besides property (4.48) they also satisfy:

$$
\boldsymbol{C}_{g_{1}, g_{2}}^{g}=\boldsymbol{C}_{g, g_{2}^{-1}}^{g_{1}}
$$

Moreover the following identities hold: 
i) deformed Jacobi identities:

$$
C_{h_{1}, g_{1}}^{k} C_{k, g_{2}}^{h_{2}}-\Lambda_{g_{1}, g_{2}}^{g_{3}, g_{4}} C_{h_{1}, g_{3}}^{k} C_{k, g_{4}}^{h_{2}}=C_{g_{1}, g_{2}}^{k} C_{h_{1}, k}^{h_{2}}
$$

ii) fusion identities:

$$
C_{h_{1}, g}^{k} \boldsymbol{C}_{k, g^{\prime}}^{h_{2}}=C_{g, g^{\prime}}^{h} \boldsymbol{C}_{h_{1}, h}^{h_{2}}
$$

Thus the $\boldsymbol{C}$ structure constants are a representation (the adjoint representation) of the tangent vectors $t$.

\section{Cartan-Maurer equations, connection and curvature}

From the definition (4.18) and eq. (4.22) we deduce the Cartan-Maurer equations:

$$
d \theta^{g}+\sum_{g_{1}, g_{2}} C^{g}{ }_{g_{1}, g_{2}} \theta^{g_{1}} \wedge \theta^{g_{2}}=0
$$

where the structure constants $C_{g_{1}, g_{2}}^{g}$ are those given in (4.47).

Parallel transport of the vielbein $\theta^{g}$ can be defined as in ordinary Lie group manifolds:

$$
\nabla \theta^{g}=-\omega_{g^{\prime}}^{g} \otimes \theta^{g^{\prime}}
$$

where $\omega_{g_{2}}^{g_{1}}$ is the connection one-form:

$$
\omega_{g_{2}}^{g_{1}}=\Gamma_{g_{3}, g_{2}}^{g_{1}} \theta^{g_{3}}
$$

Thus parallel transport is a map from $\Gamma$ to $\Gamma \otimes \Gamma$; by definition it must satisfy:

$$
\nabla(a \rho)=(d a) \otimes \rho+a \nabla \rho, \quad \forall a \in A, \rho \in \Gamma
$$

and it is a simple matter to verify that this relation is satisfied with the usual parallel transport of Riemannian manifolds. As for the exterior differential, $\nabla$ can be extended to a map $\nabla: \Gamma^{\wedge n} \otimes \Gamma \longrightarrow \Gamma^{\wedge(n+1)} \otimes \Gamma$ by defining:

$$
\nabla(\varphi \otimes \rho)=d \varphi \otimes \rho+(-1)^{n} \varphi \nabla \rho
$$

Requiring parallel transport to commute with the left and right action of $G$ means:

$$
\begin{aligned}
& \mathcal{L}_{h}\left(\nabla \theta^{g}\right)=\nabla\left(\mathcal{L}_{h} \theta^{g}\right)=\nabla \theta^{g} \\
& \mathcal{R}_{h}\left(\nabla \theta^{g}\right)=\nabla\left(\mathcal{R}_{h} \theta^{g}\right)=\nabla \theta^{a d(h) g}
\end{aligned}
$$

Recalling that $\mathcal{L}_{h}(a \rho)=\left(\mathcal{L}_{h} a\right)\left(\mathcal{L}_{h} \rho\right)$ and $\mathcal{L}_{h}\left(\rho \otimes \rho^{\prime}\right)=\left(\mathcal{L}_{h} \rho\right) \otimes\left(\mathcal{L}_{h} \rho^{\prime}\right), \quad \forall a \in$ $A, \rho, \rho^{\prime} \in \Gamma$ (and similar for $\mathcal{R}_{h}$ ), and substituting (4.56) yields respectively:

$$
\Gamma_{g_{3}, g_{2}}^{g_{1}} \in \boldsymbol{C}
$$


and

$$
\Gamma_{a d(h) g_{3}, a d(h) g_{2}}^{a d(h) g_{1}}=\Gamma_{g_{3}, g_{2}}^{g_{1}}
$$

Therefore the same situation arises as in the case of Lie groups, for which parallel transport on the group manifold commutes with left and right action iff the connection components are $\operatorname{ad}(G)$ - conserved constant tensors. As for Lie groups, condition (4.63) is satisfied if one takes $\Gamma$ proportional to the structure constants. In our case, we can take any combination of the $C$ or $\boldsymbol{C}$ structure constants, since both are $a d(G)$ conserved constant tensors. As we see below, the $C$ constants can be used to define a torsionless connection, while the $\boldsymbol{C}$ constants define a parallelizing connection.

As usual, the curvature arises from $\nabla^{2}$ :

$$
\begin{gathered}
\nabla^{2} \theta^{g}=-R_{g^{\prime}}^{g} \otimes \theta^{g^{\prime}} \\
R_{g_{2}}^{g_{1}} \equiv d \omega_{g_{2}}^{g_{1}}+\omega_{g_{3}}^{g_{1}} \wedge \omega_{g_{2}}^{g_{3}}
\end{gathered}
$$

The torsion $R^{g}$ is defined by:

$$
R^{g_{1}} \equiv d \theta^{g_{1}}+\omega_{g_{2}}^{g_{1}} \wedge \theta^{g_{2}}
$$

Using the expression of $\omega$ in terms of $\Gamma$ and the Cartan-Maurer equations yields

$$
\begin{gathered}
R_{g_{2}}^{g_{1}}=\left(-\Gamma_{h, g_{2}}^{g_{1}} C_{g_{3}, g_{4}}^{h}+\Gamma_{g_{3}, h}^{g_{1}} \Gamma_{g_{4}, g_{2}}^{h}\right) \theta^{g_{3}} \wedge \theta^{g_{4}}= \\
=\left(-\Gamma_{h_{2} g_{2}}^{g_{1}} C_{g_{3}, g_{4}}^{h}+\Gamma_{g_{3}, h}^{g_{1}} \Gamma_{g_{4}, g_{2}}^{h}-\Gamma_{g_{4}, h}^{g_{1}} \Gamma_{g_{4} g_{3} g_{4}^{-1}, g_{2}}^{h}\right) \theta^{g_{3}} \otimes \theta^{g_{4}} \\
R^{g_{1}}=\left(-C_{g_{2}, g_{3}}^{g_{1}}+\Gamma_{g_{2}, g_{3}}^{g_{1}}\right) \theta^{g_{2}} \wedge \theta^{g_{3}}= \\
\left(-C_{g_{2}, g_{3}}^{g_{1}}+\Gamma_{g_{2}, g_{3}}^{g_{1}}-\Gamma_{g_{3}, g_{3} g_{2} g_{3}^{-1}}^{g_{1}}\right) \theta^{g_{2}} \otimes \theta^{g_{3}}
\end{gathered}
$$

Thus a connection satisfying:

$$
\Gamma_{g_{2}, g_{3}}^{g_{1}}-\Gamma_{g_{3}, g_{3} g_{2} g_{3}^{-1}}^{g_{1}}=\boldsymbol{C}_{g_{2}, g_{3}}^{g_{1}}
$$

corresponds to a vanishing torsion $R^{g}=0$ and could be referred to as a "Riemannian" connection.

On the other hand, the choice:

$$
\Gamma_{g_{2}, g_{3}}^{g_{1}}=\boldsymbol{C}_{g_{3}, g_{2}^{-1}}^{g_{1}}
$$

corresponds to a vanishing curvature $R_{g^{\prime}}^{g}=0$, as can be checked by using the fusion equations (4.54) and property (4.52). Then (4.69) can be called the parallelizing connection: finite groups are parallelizable. 


\section{Tensor transformations}

Under the familiar transformation of the connection 1-form:

$$
\left(\omega^{i}{ }_{j}\right)^{\prime}=a_{k}^{i} \omega_{l}^{k}\left(a^{-1}\right)_{j}^{l}+a_{k}^{i} d\left(a^{-1}\right)_{j}^{k}
$$

the curvature 2-form transforms homogeneously:

$$
\left(R_{j}^{i}\right)^{\prime}=a_{k}^{i} R_{l}^{k}\left(a^{-1}\right)_{j}^{l}
$$

The transformation rule (4.70) can be seen as induced by the change of basis $\theta^{i}=$ $a_{j}^{i} \theta^{j}$, with $a^{i}{ }_{j}$ invertible $x$-dependent matrix (use eq. (4.58) with $a \rho=a^{i}{ }_{j} \theta^{j}$ ).

\section{Metric}

The metric tensor $\gamma$ can be defined as an element of $\Gamma \otimes \Gamma$ :

$$
\gamma=\gamma_{i, j} \theta^{i} \otimes \theta^{j}
$$

Requiring it to be invariant under left and right action of $G$ means:

$$
\mathcal{L}_{h}(\gamma)=\gamma=\mathcal{R}_{h}(\gamma)
$$

or equivalently, recalling $\mathcal{L}_{h}\left(\theta^{i} \otimes \theta^{j}\right)=\theta^{i} \otimes \theta^{j}, \mathcal{R}_{h}\left(\theta^{i} \otimes \theta^{j}\right)=\theta^{\operatorname{ad}(h) i} \otimes \theta^{\operatorname{ad}(h) j}$ :

$$
\gamma_{i, j} \in \boldsymbol{C}, \quad \gamma_{a d(h) i, a d(h) j}=\gamma_{i, j}
$$

These properties are analogous to the ones satisfied by the Killing metric of Lie groups, which is indeed constant and invariant under the adjoint action of the Lie group.

On finite $G$ there are various choices of biinvariant metrics. One can simply take $\gamma_{i, j}=\delta_{i, j}$, or $\gamma_{i, j}=\boldsymbol{C}_{l, i}^{k} \boldsymbol{C}_{k, j}^{l}$.

For any biinvariant metric $\gamma_{i j}$ there are tensor transformations $a^{i}{ }_{j}$ under which $\gamma_{i j}$ is invariant, i.e.:

$$
a_{h^{\prime}}^{h} \gamma_{h, k} a_{k^{\prime}}^{k}=\gamma_{h^{\prime}, k^{\prime}} \Leftrightarrow a_{h^{\prime}}^{h} \gamma_{h, k}=\gamma_{h^{\prime}, k^{\prime}}\left(a^{-1}\right)_{k}^{k^{\prime}}
$$

These transformations are simply given by the matrices that rotate the indices according to the adjoint action of $G$ :

$$
a_{h^{\prime}}^{h}(g)=\delta_{h^{\prime}}^{a d(\alpha(g)) h}
$$

where $\alpha(g): G \mapsto G$ is an arbitrary mapping. Then these matrices are functions of $G$ via this mapping, and their action leaves $\gamma$ invariant because of the its biinvariance (4.74). Indeed substituting these matrices in (4.75) yields:

$$
a_{h^{\prime}}^{h}(g) \gamma_{h, k} a_{k^{\prime}}^{k}(g)=\gamma_{a d\left([\alpha(g)]^{-1}\right) h^{\prime}, a d\left([\alpha(g)]^{-1}\right) k^{\prime}}=\gamma_{h^{\prime}, k^{\prime}}
$$


proving the invariance of $\gamma$.

Consider now a contravariant vector $\varphi^{i}$ transforming as $\left(\varphi^{i}\right)^{\prime}=a_{j}^{i}\left(\varphi^{j}\right)$. Then using (4.75) one can easily see that

$$
\left(\varphi^{k} \gamma_{k, i}\right)^{\prime}=\varphi^{k^{\prime}} \gamma_{k^{\prime}, i^{\prime}}\left(a^{-1}\right)^{i^{\prime}}{ }_{i}
$$

i.e. the vector $\varphi_{i} \equiv \varphi^{k} \gamma_{k, i}$ indeed transforms as a covariant vector.

\section{Lie derivative and diffeomorphisms}

The notion of diffeomorphisms, or general coordinate transformations, is fundamental in gravity theories. Is there such a notion in the setting of differential calculi on finite groups ? The answer is affirmative, and is based on general results obtained for Hopf algebras [19, 21, 52], of which finite groups are a simple example. As for differentiable manifolds, it relies on the existence of the Lie derivative.

Let us review the situation for Lie group manifolds. The Lie derivative $l_{t_{i}}$ along a left-invariant tangent vector $t_{i}$ is related to the infinitesimal right translations generated by $t_{i}$ :

$$
l_{t_{i}} \rho=\lim _{\varepsilon \rightarrow 0} \frac{1}{\varepsilon}\left[\mathcal{R}_{\exp \left[\varepsilon t_{i}\right]} \rho-\rho\right]
$$

$\rho$ being an arbitrary tensor field. Introducing the coordinate dependence

$$
l_{t_{i}} \rho(y)=\lim _{\varepsilon \rightarrow 0} \frac{1}{\varepsilon}\left[\rho\left(y+\varepsilon t_{i}\right)-\rho(y)\right]
$$

identifies the Lie derivative $l_{t_{i}}$ as a directional derivative along $t_{i}$. Note the difference in meaning of the symbol $t_{i}$ in the r.h.s. of these two equations: a group generator in the first, and the corresponding tangent vector in the second.

For finite groups the Lie derivative takes the form:

$$
l_{t_{g}} \rho=\left[\mathcal{R}_{g^{-1}} \rho-\rho\right]
$$

so that the Lie derivative along $t_{g}$ coincides with the tangent vector $t_{g}$ :

$$
l_{t_{g}}=\mathcal{R}_{g^{-1}}-i d=t_{g}
$$

cf. the definition of $t_{g}$ in (4.45). For example

$$
l_{t_{g}}\left(\theta^{g_{1}} \otimes \theta^{g_{2}}\right)=\theta^{a d\left(g^{-1}\right) g_{1}} \otimes \theta^{a d\left(g^{-1}\right) g_{2}}-\theta^{g_{1}} \otimes \theta^{g_{2}}
$$

As in the case of differentiable manifolds, the Cartan formula for the Lie derivative acting on p-forms holds:

$$
l_{t_{g}}=i_{t_{g}} d+d i_{t_{g}}
$$

see ref.s 19, 52, 47, 48.

Exploiting this formula, diffeomorphisms (Lie derivatives) along generic tangent vectors $V$ can also be consistently defined via the operator:

$$
l_{V}=i_{V} d+d i_{V}
$$


This requires a suitable definition of the contraction operator $i_{V}$ along generic tangent vectors $V$, discussed in ref. [52, 47].

We have then a way of defining "diffeomorphisms" along arbitrary (and xdependent) tangent vectors for any tensor $\rho$ :

$$
\delta \rho=l_{V} \rho
$$

and of testing the invariance of candidate lagrangians under the generalized Lie derivative.

\section{Haar measure and integration}

Since we want to define actions (integrals on $p$-forms), we must now define integration of $p$-forms on finite groups.

Let us start with integration of functions $f$. We define the integral map $h$ as a linear functional $h: F u n(G) \mapsto C$ satisfying the left and right invariance conditions:

$$
h\left(\mathcal{L}_{g} f\right)=0=h\left(\mathcal{R}_{g} f\right)
$$

Then this map is uniquely determined (up to a normalization constant), and is simply given by the "sum over $G$ " rule:

$$
h(f)=\sum_{g \in G} f(g)
$$

Next we turn to define the integral of a p-form. Within the differential calculus we have a basis of left-invariant 1-forms, which may allow the definition of a biinvariant volume element. In general for a differential calculus with $n$ independent tangent vectors, there is an integer $p \geq n$ such that the linear space of $p$-forms is 1-dimensional, and $(p+1)$ - forms vanish identically 3 . This means that every product of $p$ basis one-forms $\theta^{g_{1}} \wedge \theta^{g_{2}} \wedge \ldots \wedge \theta^{g_{p}}$ is proportional to one of these products, that can be chosen to define the volume form vol:

$$
\theta^{g_{1}} \wedge \theta^{g_{2}} \wedge \ldots \wedge \theta^{g_{p}}=\epsilon^{g_{1}, g_{2}, \ldots g_{p}} v o l
$$

where $\epsilon^{g_{1}, g_{2}, \ldots g_{p}}$ is the proportionality constant. Note that the volume $p$-form is obviously left invariant. We can prove that it is also right invariant with the following argument. Suppose that $v o l$ be given by $\theta^{h_{1}} \wedge \theta^{h_{2}} \wedge \ldots \wedge \theta^{h_{p}}$ where $h_{1}, h_{2}, \ldots h_{p}$ are given group element labels. Then the right action on vol yields:

$$
\mathcal{R}_{g}\left[\theta^{h_{1}} \wedge \ldots \wedge \theta^{h_{p}}\right]=\theta^{a d(g) h_{1}} \wedge \ldots \wedge \theta^{a d(g) h_{p}}=\epsilon^{a d(g) h_{1}, \ldots a d(g) h_{p}} v o l
$$

Recall now that the "epsilon tensor" $\epsilon$ is necessarily made out of products of the $\Lambda$ tensor of eq. (4.25), defining the wedge product. This tensor is invariant under the adjoint action $\operatorname{ad}(g)$, and so is the $\epsilon$ tensor. Therefore $\epsilon^{a d(g) h_{1}, \ldots a d(g) h_{p}}=\epsilon^{h_{1}, \ldots h_{p}}=1$

\footnotetext{
${ }^{3}$ with the exception of $Z_{2}$, see Section 4.2
} 
and $\mathcal{R}_{g}$ vol $=$ vol. This can be verified in the $S_{3}$ example of [47, 48], and in the $Z_{2}$ case of next Section.

Having identified the volume $p$-form it is natural to set

$$
\int f v o l \equiv h(f)=\sum_{g \in G} f(g)
$$

and define the integral on a $p$-form $\rho$ as:

$$
\begin{aligned}
& \int \rho=\int \rho_{g_{1}, \ldots g_{p}} \theta^{g_{1}} \wedge \ldots \wedge \theta^{g_{p}}= \\
& \int \rho_{g_{1}, \ldots g_{p}} \epsilon^{g_{1}, \ldots g_{p}} v o l \equiv \\
& \equiv \sum_{g \in G} \rho_{g_{1}, \ldots g_{p}}(g) \epsilon^{g_{1}, \ldots g_{p}}
\end{aligned}
$$

Due to the biinvariance of the volume form, the integral map $\int: \Gamma^{\wedge p} \mapsto \boldsymbol{C}$ satisfies the biinvariance conditions:

$$
\int \mathcal{L}_{g} f=\int f=\int \mathcal{R}_{g} f
$$

Moreover, under the assumption that $d\left(\theta^{g_{2}} \wedge \ldots \wedge \theta^{g_{p}}\right)=0$, i.e. that any exterior product of $p-1$ left-invariant one-forms $\theta$ is closed, the important property holds:

$$
\int d f=0
$$

with $f$ any $(p-1)$-form: $f=f_{g_{2}, \ldots g_{p}} \theta^{g_{2}} \wedge \ldots \wedge \theta^{g_{p}}$. This property, which allows integration by parts, has a simple proof. Rewrite $\int d f$ as:

$$
\begin{aligned}
& \int d f=\int\left(d f_{g_{2}, \ldots g_{p}}\right) \theta^{g_{2}} \wedge \ldots \wedge \theta^{g_{p}}+ \\
& +\int f_{g_{2}, \ldots g_{p}} d\left(\theta^{g_{2}} \wedge \ldots \wedge \theta^{g_{p}}\right)
\end{aligned}
$$

The second term in the r.h.s. vanishes by assumption. Using now (4.42) and (4.91):

$$
\begin{aligned}
& \int d f=\int\left(t_{g_{1}} f_{g_{2}, \ldots g_{p}}\right) \theta^{g_{1}} \wedge \theta^{g_{2}} \wedge \ldots \wedge \theta^{g_{p}}= \\
& \int\left[\mathcal{R}_{g_{1}^{-1}} f_{g_{2}, \ldots g_{p}}-f_{g_{2}, \ldots g_{p}}\right] \epsilon^{g_{1}, \ldots g_{p}} v o l= \\
& \epsilon^{g_{1}, \ldots g_{p}} \sum_{g \in G}\left[\mathcal{R}_{g_{1}^{-1}} f_{g_{2}, \ldots g_{p}}(g)-f_{g_{2}, \ldots g_{p}}(g)\right]= \\
& =0
\end{aligned}
$$

Q.E.D. 


\subsection{Bicovariant calculus on $Z_{2}$}

In this Section we illustrate the general theory on $Z_{2}$, the simplest possible example.

Elements: $e, u$

with $u^{2}=e$.

Conjugation classes: $\{e\},\{u\}$.

There is therefore only one bicovariant calculus, corresponding to the only nontrivial congugation class $\{u\}$, of dimension 1 .

Basis of functions on $Z_{2}:\left\{x^{e}, x^{u}\right\}$. Any function $f$ can be expanded as: $f=$ $f_{e} x^{e}+f_{u} x^{u}$

The left action of $Z_{2}$ on the functions is simply: $\mathcal{L}_{e}=i d, \mathcal{L}_{u}\left(x^{e}\right)=x^{u}, \mathcal{L}_{u}\left(x^{u}\right)=x^{e}$. The right action coincides with the left action since $Z_{2}$ is abelian.

Partial derivatives:

$$
d f=\sum_{g \in G} f_{g} d x^{g}=\sum_{g \neq e} f_{g} d x^{g}+f_{e} d x^{e}=\left(f_{u}-f_{e}\right) d x^{u} \Rightarrow \partial_{u} f=f_{u}-f_{e}
$$

Left-invariant one-forms:

$$
\begin{aligned}
& \theta^{u}=x^{u} d x^{e}+x^{e} d x^{u}=\left(x^{e}-x^{u}\right) d x^{u} \\
& \left(\theta^{e}=x^{e} d x^{e}+x^{u} d x^{u}=\left(x^{u}-x^{e}\right) d x^{u}=-\theta^{u}\right)
\end{aligned}
$$

Inversion formula:

$$
d x^{u}=\left(x^{e}-x^{u}\right) \theta^{u}, \quad\left(d x^{e}=-d x^{u}\right)
$$

Commutations $x, \theta$ :

$$
f \theta^{u}=\theta^{u} \mathcal{R}_{u} f \Rightarrow x^{e} \theta^{u}=\theta^{u} x^{u}, \quad x^{u} \theta^{u}=\theta^{u} x^{e}
$$

Commutations $x, d x$ :

$$
x^{e} d x^{u}=d x^{u} x^{u}, \quad x^{u} d x^{u}=d x^{u} x^{e} \Rightarrow f d x^{u}=d x^{u} \mathcal{R}_{u} f
$$

Left and right action on $\theta^{u}$ :

$$
\mathcal{L}_{u} \theta^{u}=\mathcal{R}_{u} \theta^{u}=\left(x^{u}-x^{e}\right) d x^{e}=\theta^{u}
$$

Exterior product:

$$
\theta^{u} \wedge \theta^{u}=0
$$

when using the general formula (4.25). However the case of $Z_{2}$ is special in this respect: $\theta^{u} \wedge \theta^{u}$ can be set different from zero consistently with the differential 
calculus. Indeed for $Z_{2}$ (and only for this case) the whole calculus is consistent with the exterior product:

$$
\theta^{u} \wedge \theta^{u}=\theta^{u} \otimes \theta^{u}
$$

For example taking the exterior derivative of both members of the commutation relations $x^{u} d x^{u}=d x^{u} x^{e}$ yields an identity (using $d^{2}=0$ and $d x^{e}=-d x^{u}$ ), and does not imply $d x^{u} \wedge d x^{u}=0$. Using then the expression of $d x^{u}$ in terms of $\theta^{u}$ and the $x, \theta$ commutations, one finds

$$
d x^{u} \wedge d x^{u}=-\theta^{u} \wedge \theta^{u}
$$

so that $\theta^{u} \wedge \theta^{u}$ can be different from zero. In the case of $Z_{N}, N>2$ the situation is different since taking the exterior derivative of the $x, d x$ commutations implies the vanishing of the exterior product of a left-invariant one-form with itself: then one has to adopt the canonical definition as given in (4.25).

For $Z_{2}$, we denote the two possibilities calculus I $\left(d x^{u} \wedge d x^{u}=0\right)$ and calculus II $\left(d x^{u} \wedge d x^{u} \neq 0\right)$.

Tangent vector

$t_{u} f=\left(\mathcal{R}_{u}-i d\right) f, \quad t_{u} t_{u}=\left(\mathcal{R}_{u}-i d\right)\left(\mathcal{R}_{u}-i d\right)=\mathcal{R}_{e}-2 \mathcal{R}_{u}+i d=2\left(i d-\mathcal{R}_{u}\right)=-2 t_{u}$

\section{Cartan-Maurer equations}

Calculus I:

$$
d \theta^{u}=0
$$

Calculus II:

$$
d \theta^{u}=d x^{u} \wedge d x^{e}+d x^{e} \wedge d x^{u}=-2 d x^{u} \wedge d x^{u}=2 \theta^{u} \wedge \theta^{u}
$$

Connection

$$
\omega^{u}{ }_{u}=\Gamma_{u, u}^{u} \theta^{u}
$$

where $\Gamma_{u, u}^{u}=$ constant $=c$ satisfies left and right invariance.

Curvature and torsion

Calculus I:

$$
\begin{aligned}
& R^{u}{ }_{u}=d \omega^{u}{ }_{u}+\omega^{u}{ }_{u} \wedge \omega^{u}{ }_{u}=c d \theta^{u}+c^{2} \theta^{u} \wedge \theta^{u}=0 \\
& T^{u}=d \theta^{u}+c \theta^{u} \wedge \theta^{u}=0
\end{aligned}
$$

Calculus II:

$$
\begin{aligned}
& R_{u}^{u}=c d \theta^{u}+c^{2} \theta^{u} \wedge \theta^{u}=\left(2 c+c^{2}\right) \theta^{u} \wedge \theta^{u} \\
& T^{u}=d \theta^{u}+c \theta^{u} \wedge \theta^{u}=(2+c) \theta^{u} \wedge \theta^{u}
\end{aligned}
$$

In this case $c=-2$ gives a flat and torsionless connection. 


\section{Integration}

For calculus I, the volume form is the one-form $\theta^{u}$, and the integral of a one-form $\rho=\rho_{u} \theta^{u}$ is simply:

$$
\int \rho=\int \rho_{u} \theta^{u}=\int \rho_{u} v o l=\sum_{g \in G} \rho_{u}(g)=\rho_{u}(e)+\rho_{u}(u)
$$

Integration by parts holds since:

$$
\int d f=\int\left(t_{u} f\right) \theta^{u}=\int\left[\left(\mathcal{R}_{u}-i d\right) f\right] v o l=\sum_{g \in G}\left(\mathcal{R}_{u} f-f\right)(g)=0
$$

for $f=0$-form.

In the special case of $Z_{2}$, choosing calculus II, there is no upper limit to the degree of a $p$-form, since all the products $\theta^{u} \wedge \theta^{u} \wedge \ldots \theta^{u}$ are nonvanishing. Then any one of these products, being bi-invariant, can be chosen as volume form ! Supposing to take the $p$-form volume as volume form, the integral of a p-form $\rho_{u, u, \ldots u} \theta^{u} \wedge \theta^{u} \wedge \ldots \theta^{u}$ is then simply $\rho_{u, u, \ldots u}(e)+\rho_{u, u, \ldots u}(u)$. Choosing $\theta^{u} \wedge \theta^{u}$ as volume form, we find $\int d \sigma \neq 0$ (where $\sigma$ is a 1 form); indeed:

$$
\int d \sigma=\int d\left(\sigma_{u} \theta^{u}\right)=\int\left(t_{u} \sigma_{u}\right) \theta^{u} \wedge \theta^{u}+2 \int \sigma_{u} \theta^{u} \wedge \theta^{u}=2\left[\sigma_{u}(e)+\sigma_{u}(u)\right]
$$

Note 4.3: Choosing higher volume forms one retrieves the integration by parts rule, essentially because an exterior product of two or more $\theta^{u}$ 's is closed.

\subsection{Kaluza-Klein gauge theory on $M_{4} \times Z_{2}$}

In this example we label the $M_{4}$ coordinates as $x^{\mu}$ and the $Z_{2}$ coordinate as $y$. Field theories (and in particular gauge theories) on discrete spaces have been considered by many authors. The treatment of this Section is closer in spirit to the works of [53, 50, 54, 55,

\section{Calculus on $M_{4} \times Z_{2}$}

The $y$ coordinate can take the values $e, u$, and any function $f$ on $M_{4} \times Z_{2}$ is expanded as:

$$
f(x, y)=f_{e}(x) y^{e}+f_{u}(x) y^{u}
$$

where $y^{e}, y^{u}$ are defined as usual to be "dual" to the $Z_{2}$ points: $y^{e}(e)=y^{u}(u)=$ $1, y^{e}(u)=y^{u}(e)=0$. We will frequently use the notation:

$$
\tilde{f} \equiv \mathcal{R}_{u} f=f_{u}(x) y^{e}+f_{e}(x) y^{u}
$$

Thus $\tilde{f}$ is obtained from $f$ simply by exchanging its components along $y^{e}, y^{u}$. 
The only independent $Z_{2}$ differential $d y^{u}$ will be simply denoted by $d y$.

Note that

$$
\tilde{f} d y=d y f
$$

cf. eq. (4.102).

To define completely the differential geometry on $M_{4} \times Z_{2}$ we need the rules:

$$
d x^{\mu} \wedge d y=-d y \wedge d x^{\mu}, \quad f d x^{\mu}=d x^{\mu} f
$$

A basis of differentials is given by $d x^{M}=\left(d x^{\mu}, d y\right)$, so that any one-form $A(x, y)$ is expanded as:

$$
A(x, y)=A(x, y)_{M} d x^{M}=A_{\mu}(x, y) d x^{\mu}+A_{\bullet}(x, y) d y
$$

Finally, integration of a function $f(x, y)$ on $M_{4} \times Z_{2}$ is defined by:

$$
\int_{M_{4} \times Z_{2}} f \text { vol } \equiv \int_{M_{4}} \sum_{Z_{2}} f(x, y) d^{4} x=\int_{M_{4}}\left[f_{e}(x)+f_{u}(x)\right] d^{4} x
$$

\section{Gauge potential}

Consider now the one-form $A$ to be the potential 1-form of a gauge theory: then it must be also matrix valued. For example in ordinary Yang-Mills theory, $A(x)=$ $A_{\mu}^{I} T_{I} d x^{\mu}$ where $T_{I}$ are the generators of the gauge group $\mathrm{G}$ in some irreducible representation.

As in the usual case, we define G-gauge transformations on the potential $A(x, y)$ as:

$$
A^{\prime}=-(d G) G^{-1}+G A G^{-1}
$$

where $G=G(x, y)$ is a group element is some irrep, depending on the point $(x, y) \in$ $M_{4} \times Z_{2}$. In components:

$$
A_{\mu}^{\prime}=-\left(\partial_{\mu} G\right) G^{-1}+G A_{\mu} G^{-1}, \quad A_{\bullet}^{\prime}=-\left(\partial_{\bullet} G\right) \tilde{G}^{-1}+G A_{\bullet} \tilde{G}^{-1}
$$

the derivative along $y$ being denoted by $\partial_{\bullet}$. Note that

$$
\partial_{\bullet} f(x, y)=f_{u}(x)-f_{e}(x)=\frac{1}{2}\left(y^{e}-y^{u}\right)(\tilde{f}-f) \equiv J(\tilde{f}-f)
$$

where we have introduced the function $J \equiv \frac{1}{2}\left(y^{e}-y^{u}\right)$.

The transformation laws tell us something about the matrix structure of the gauge potential $A$. The potential components $A_{\mu}$ must belong to the Lie algebra of $\mathrm{G}$, since $\left(\partial_{\mu} G\right) G^{-1} \in \operatorname{Lie}(G)$. On the other hand $A_{\bullet}$ does not belong to $\operatorname{Lie}(\mathrm{G})$ but rather to the group algebra of $\mathrm{G}$. Indeed $\partial_{\bullet} G$ is a finite difference of group elements, and thus $\left(\partial_{\bullet} G\right) G^{-1}$ belongs to the group algebra; then the second eq. in (4.125) implies that $A_{\text {}}$ is matrix valued in the group algebra of $\mathrm{G}$. 
For definiteness, we consider unitary groups, so that $G^{\dagger}=G^{-1}$. Then $A_{\mu}$ is antihermitian (since the generators $T_{I}$ are antihermitian), while $A_{\bullet}$, being in the $U(N)$ group algebra is a sum of $U(N)$ matrices.

We can consistently incorporate hermitian conjugation in the $M_{4} \times Z_{2}$ - differential calculus by setting:

$$
\begin{aligned}
& \left(d x^{\mu}\right)^{\dagger}=d x^{\mu}, \quad(d y)^{\dagger}=d y \\
& (f d y)^{\dagger}=d y f^{\dagger}
\end{aligned}
$$

\section{Matter fields}

Matter fields $\psi$ are taken to transform in an irrep of G:

$$
\psi^{\prime}=G \psi, \quad\left(\psi^{\dagger}\right)^{\prime}=\psi^{\dagger} G^{\dagger}=\psi^{\dagger} G^{-1}
$$

and their covariant derivative, defined by

$$
D \psi=d \psi+A \psi, \quad D \psi^{\dagger}=d \psi^{\dagger}-\psi^{\dagger} A
$$

transforms as it should: $(D \psi)^{\prime}=G(D \psi),\left(D \psi^{\dagger}\right)^{\prime}=\left(D \psi^{\dagger}\right) G^{-1}$. Requiring compatibility of hermitian conjugation with the covariant derivative, i.e. $(D \psi)^{\dagger}=D \psi^{\dagger}$, implies:

$$
A^{\dagger}=-A
$$

that is, $A$ must be an antihermitian connection. This is compatible with its transformation rule (4.124). In components the antihermitian condition reads:

$$
A_{\mu}^{\dagger}=-A_{\mu}, \quad A_{\bullet}^{\dagger}=-\tilde{A}_{\bullet}
$$

\section{Field strength}

The field strength $F$ is formally defined as usual:

$$
F=d A+A \wedge A
$$

so that it transforms as:

$$
F^{\prime}=G F G^{-1}
$$

The components of the 2-form $F$ are labelled as follows:

$$
F \equiv F_{M N} d x^{M} \wedge d x^{N} \equiv F_{\mu \nu} d x^{\mu} \wedge d x^{\nu}+2 F_{\mu \bullet} d x^{\mu} \wedge d y+F_{\bullet \bullet} d y \wedge d y
$$

Therefore the $F$ components are given by:

$$
\begin{aligned}
F_{\mu \nu} & =\frac{1}{2}\left(\partial_{\mu} A_{\nu}-\partial_{\nu} A_{\mu}+A_{\mu} A_{\nu}-A_{\nu} A_{\mu}\right) \\
F_{\mu \bullet} & =\frac{1}{2}\left(\partial_{\mu} A_{\bullet}-\partial_{\bullet} A_{\mu}+A_{\mu} A_{\bullet}-A_{\bullet} \tilde{A}_{\mu}\right) \\
F_{\bullet \bullet} & =\partial_{\bullet} A_{\bullet}+A_{\bullet} \tilde{A}_{\bullet}
\end{aligned}
$$


and transform as:

$$
\begin{aligned}
& F_{\mu \nu}^{\prime}(x, y)=G(x, y) F_{\mu \nu}(x, y) G^{-1}(x, y) \\
& F_{\mu \bullet}^{\prime}(x, y)=G(x, y) F_{\mu \bullet}(x, y) \tilde{G}^{-1}(x, y) \\
& F_{\bullet \bullet}^{\prime}(x, y)=G(x, y) F_{\bullet \bullet}(x, y) G^{-1}(x, y)
\end{aligned}
$$

\section{The gauge action}

Formally the gauge action has the same expression as in the usual case:

$$
A_{Y M}=\int_{M_{4} \times Z_{2}} \operatorname{Tr}_{G}\left[F_{A B} F_{A B}^{\dagger}\right] \text { vol }
$$

When expanded into components:

$$
A_{Y M}=\int_{M_{4} \times Z_{2}} \sum_{Z_{2}} \operatorname{Tr}_{G}\left[F_{\mu \nu} F_{\mu \nu}^{\dagger}+2 F_{\mu \bullet} F_{\mu \bullet}^{\dagger}+F_{\bullet \bullet} F_{\bullet \bullet}^{\dagger}\right] d^{4} x
$$

This action is invariant under the $\mathrm{G}$ gauge transformations (4.141). We now rewrite it in a suggestive way, by introducing the "link" field $U(x, y)$ :

$$
U(x, y) \equiv \mathbb{I}+J^{-1} A \bullet
$$

Then

$$
F_{\mu \bullet}=\frac{1}{2} J\left(\partial_{\mu} U+A_{\mu} U-U \tilde{A}_{\mu}\right) \equiv \frac{1}{2} J D_{\mu} U, \quad F_{\bullet \bullet}=\frac{1}{4}(1 I-U \tilde{U})
$$

Using the transformation rules (4.125) one finds that the link field $U$ and its covariant derivative vary homogeneously:

$$
U^{\prime}=G U \tilde{G}^{-1}, \quad\left(D_{\mu} U\right)^{\prime}=G\left(D_{\mu} U\right) \tilde{G}^{-1}
$$

Moreover the antihermiticity of $A(4.132)$ implies:

$$
U^{\dagger}=\tilde{U}
$$

(use $\tilde{J}=-J)$. Expanding $U(x, y)$ as $U_{e}(x) y^{e}+U_{u}(x) y^{u}$, relation 4.147) becomes $U_{e}^{\dagger}=U_{u}$.

Using the expressions (4.145) for the field strength components finally yields the action in the form:

$$
A_{Y M}=\int d^{4} x \operatorname{Tr}_{G} \sum_{Z_{2}}\left[F_{\mu \nu} F_{\mu \nu}+\frac{1}{16} D_{\mu} U\left(D_{\mu} U\right)^{\dagger}+\frac{1}{16}\left(\mathbb{I}-U U^{\dagger}\right)^{2}\right]
$$

The sum on $Z_{2}$ is easy to perform, and taking into account $U_{e}^{\dagger}=U_{u}$ we find:

$$
A_{Y M}=2 \int d^{4} x \operatorname{Tr}_{G}\left[F_{\mu \nu} F_{\mu \nu}+\frac{1}{16} D_{\mu} U\left(D_{\mu} U\right)^{\dagger}+\frac{1}{16}\left(\mathbb{I}-U U^{\dagger}\right)^{2}\right]
$$


where now $U(x) \equiv U_{e}(x)$ can be seen as a complex Higgs field, with a symmetrybreaking potential. The cyclic property of $\operatorname{Tr}_{G}$ has been used to achieve this final form of $A_{Y M}$. Moreover we have identified for simplicity $A_{\mu} \equiv A_{\mu}(u)=A_{\mu}(e)$ so that the sum on $Z_{2}$ of the usual Yang-Mills term just gives a factor of 2 .

\section{Coupling to fermion matter}

We can add a Dirac term $\mathcal{L}_{\text {Dirac }}$ to the integrand of $A_{Y M}$ :

$$
\mathcal{L}_{\text {Dirac }}=\operatorname{Re}\left[i \psi^{\dagger} \gamma_{0} \gamma_{M} D_{M} \psi\right]
$$

where now the matter field $\psi(x, y)$ is a $d=5$ Dirac spinor and has therefore 4 complex spinor components. Splitting the sum on the index M:

$$
\mathcal{L}_{\text {Dirac }}=\operatorname{Re}\left[i \psi^{\dagger} \gamma_{0} \gamma_{\mu} D_{\mu} \psi\right]+\operatorname{Re}\left[i \psi^{\dagger} \gamma_{0} \gamma_{5} \partial_{\bullet} \psi+i \psi^{\dagger} \gamma_{0} \gamma_{5} A \cdot \tilde{\psi}\right]
$$

The first term is just the usual kinetic term in $d=4$; the last two terms give:

$$
\operatorname{Re}\left[-i J \bar{\psi} \gamma_{5} \psi+i J \bar{\psi} \gamma_{5} U \tilde{\psi}\right]
$$

The first term in square parentheses disappears (since its real part vanishes) and the second is:

$$
R e\left[i \bar{\psi}_{e} \gamma_{5} U_{e} \psi_{u} y^{e}-i \bar{\psi}_{u} \gamma_{5} U_{u} \psi_{e} y^{u}\right]
$$

Summing on $Z_{2}$ and redefining $\psi \equiv \psi_{e}, \chi \equiv i \gamma_{5} \psi_{u}, \quad U \equiv U_{e}$ one finds finally:

$$
\mathcal{L}_{\text {Dirac }}=i\left(\bar{\psi} \gamma_{\mu} D_{\mu} \psi+\bar{\chi} \gamma_{\mu} D_{\mu} \chi\right)+\bar{\psi} U \chi+\bar{\chi} U^{\dagger} \psi
$$

that is a kinetic term for the Dirac fields, and Yukawa couplings Higgs-Fermi-Fermi. We emphasize the appearance of the correct Higgs couplings to the Fermi fields as an output of the Kaluza-Klein mechanism on $M_{4} \times Z_{2}$ rather than an ad hoc addition to the Lagrangian. Also, the Higgs sector appears in (4.149) with the right form of the potential. This provides a nice interpretation of the Higgs appearance in the $d=4$ theory in terms of a Kaluza-Klein gauge theory coupled to Dirac fermions on $M_{4} \times Z_{2}$. The Higgs field is the component of the potential 1-form along the discrete dimension.

Note that the Kaluza-Klein mechanism on discrete internal spaces yields a finite number of fields in $d=4$ : there is no infinite tower of massive modes ! The "harmonic" analysis (4.1) on finite groups is elementary.

\section{Acknowledgements}

I have benefited from numerous discussions with G. Arcioni, P. Aschieri, F. Lizzi and M. Tarlini. 


\section{References}

[1] P.A.M. Dirac, The fundamental equations of quantum mechanics, Proc. Roy. Soc. A109 (1926) 642; On quantum algebras, Proc. Camb. Phil. Soc. 23 (1926) 412 .

[2] H.S. Snyder, Quantized space-time, Phys. Rev. 71 (1947) 38; The electromagnetic field in quantized space-time, Phys. Rev. 72 (1947) 68.

[3] E. Witten, "Noncommutative Geometry And String Field Theory," Nucl. Phys. B268, 253 (1986).

[4] A. Connes, M. R. Douglas and A. Schwarz, "Noncommutative geometry and matrix theory: Compactification on tori," JHEP 9802, 003 (1998) hepth/9711162.

[5] M. R. Douglas and C. Hull, "D-branes and the noncommutative torus," JHEP 9802, 008 (1998) hep-th/9711165.

[6] Y. E. Cheung and M. Krogh, "Noncommutative geometry from 0-branes in a background B-field," Nucl. Phys. B528, 185 (1998) hep-th/9803031.

[7] G. Landi, F. Lizzi and R. J. Szabo, "String geometry and the noncommutative torus," Commun. Math. Phys. 206, 603 (1999) hep-th/9806099].

[8] C. Chu and P. Ho, "Noncommutative open string and D-brane," Nucl. Phys. B550, 151 (1999) hep-th/9812219; "Constrained quantization of open string in background B field and noncommutative D-brane," Nucl. Phys. B568, 447 (2000) hep-th/9906192.

[9] V. Schomerus, "D-branes and deformation quantization," JHEP 9906, 030 (1999) hep-th/9903205.

[10] F. Ardalan, H. Arfaei and M. M. Sheikh-Jabbari, "Mixed branes and M(atrix) theory on noncommutative torus," hep-th/9803067; "Noncommutative geometry from strings and branes," JHEP 9902, 016 (1999) hep-th/9810072; "Dirac quantization of open strings and noncommutativity in branes," hepth/9906161.

[11] N. Seiberg and E. Witten, "String theory and noncommutative geometry," JHEP 9909, 032 (1999) hep-th/9908142.

[12] D. Bigatti, TMR lectures at Graduate school on contemporary String Theory and Brane Physics, January 2000, Torino, Italy; D. Bigatti and L. Susskind, "Magnetic fields, branes and noncommutative geometry," hep-th/9908056.

[13] M. R. Douglas, "Two lectures on D-geometry and noncommutative geometry," hep-th/9901146. 
[14] A. Connes, Non-commutative Geometry, Academic Press (1994); Non commutative geometry and Physics, IHES/M/93/32.

[15] G. Landi, "An introduction to noncommutative spaces and their geometry," hep-th/9701078.

[16] J. C. Varilly, "An introduction to noncommutative geometry," physics/9709045.

[17] J. Madore, An Introduction to Noncommutative Differential Geometry and its Physical Applications, 2nd Edition, Cambridge Univ. Press, 1999.

[18] A. Connes, Noncommutative geometry and reality, J.Math.Phys. 36 (1995) 6194; A. Connes and J. Lott, Particle models and noncommutative geometry, Nucl.Phys.Proc.Suppl. 18B (1991) 29.

[19] P. Aschieri and L. Castellani, "An Introduction to noncommutative differential geometry on quantum groups," Int. J. Mod. Phys. A8, 1667 (1993) hepth/9207084.

[20] L. Castellani, Gauge theories of quantum groups,, Phys. Lett. B292, 93 (1992), hep-th/9205103.

[21] L. Castellani, Differential calculus on $\operatorname{ISO}_{q}(N)$, quantum Poincaré algebra and q-gravity, Comm. Math. Phys. 171 (1995) 383, hep-th 9312179; The lagrangian of q-Poincaré gravity, Phys. Lett. B327 (1994) 22, hep-th 9402033.

[22] M. Kontsevich, "Deformation quantization of Poisson manifolds, I," @$\operatorname{alg} / 9709040$.

[23] B.V. Fedosov, A simple geometrical construction of deformation quantization, J.Diff.Geom. 40 (1994) 213.

[24] J.E. Moyal, Quantum mechanics as a statistical theory, Proc. Cambridge Phil. Soc., 45 (1949) 99.

[25] U. Uhlhorn, On the connection between transformations in classical mechanics and in quantum mechanics and the phase space representation of quantum mechanics, Ark. f. Fys., 11 (1956) 487.

[26] H. Groenewold, Physica 12 (1946) 405.

[27] J. von Neumann, Math. Ann. 104 (1931) 570.

[28] L. Castellani, "Quantization Rules And Dirac's Correspondence," Nuovo Cim. A48, 359 (1978); "On Canonical Transformations And Quantization Rules," Nuovo Cim. A50, 209 (1979). 
[29] T. Filk, "Divergences in a field theory on quantum space," Phys. Lett. B376, $53(1996)$.

[30] J. C. Varilly and J. M. Gracia-Bondia, "On the ultraviolet behaviour of quantum fields over noncommutative manifolds," Int. J. Mod. Phys. A14, 1305 (1999) hep-th/9804001.

[31] M. Chaichian, A. Demichev and P. Presnajder, "Quantum field theory on noncommutative space-times and the persistence of ultraviolet divergences," Nucl. Phys. B567, 360 (2000) [hep-th/9812180]; "Quantum field theory on the noncommutative plane with E(q)(2) symmetry," J. Math. Phys. 41, 1647 (2000) hep-th/9904132.

[32] M. M. Sheikh-Jabbari, "Renormalizability of the supersymmetric Yang-Mills theories on the noncommutative torus," JHEP 9906, 015 (1999) hepth/9903107].

[33] C. P. Martin and D. Sanchez-Ruiz, "The one-loop UV divergent structure of U(1) Yang-Mills theory on noncommutative $R^{4}$," Phys. Rev. Lett. 83, 476 (1999) hep-th/9903077.

[34] T. Krajewski and R. Wulkenhaar, "Perturbative quantum gauge fields on the noncommutative torus," hep-th/9903187.

[35] S. Cho, R. Hinterding, J. Madore and H. Steinacker, "Finite field theory on noncommutative geometries," hep-th/9903239.

[36] S. Minwalla, M. Van Raamsdonk and N. Seiberg, "Noncommutative perturbative dynamics," hep-th/9912072.

[37] G. Arcioni and M. A. Vazquez-Mozo, "Thermal effects in perturbative noncommutative gauge theories," JHEP 0001, 028 (2000) hep-th/9912140.

[38] A. Matusis, L. Susskind and N. Toumbas, "The IR/UV connection in the noncommutative gauge theories," hep-th/0002075.

[39] D. Fairlie, P. Fletcher and C. Zachos, "Infinite dimensional Algebras and a Trigonometric Basis for the Classical Lie Algebras", J. Math. Phys. 31 (1990) 1088.

[40] M. Alishahiha, Y. Oz and M. M. Sheikh-Jabbari, "Supergravity and large N noncommutative field theories," JHEP 9911, 007 (1999) hep-th/9909215.

[41] B. Morariu and B. Zumino, "Super Yang-Mills on the noncommutative torus," hep-th/9807198.

[42] S. Ferrara and M. A. Lledo, "Some aspects of deformations of supersymmetric field theories," JHEP 0005, 008 (2000) hep-th/0002084. 
[43] F. Antonsen, "Deformation quantisation of gravity," gr-qc/9712012.

[44] H. Garcia-Compean, J. F. Plebanski, M. Przanowski and F. J. Turrubiates, "Deformation quantization of bosonic strings," hep-th/0002212.

[45] K. Bresser, F. Müller-Hoissen, A. Dimakis and A. Sitarz, Noncommutative geometry of finite groups, J.Phys.A29 (1996) 2705, q-alg/9509004.

[46] F. Bonechi, R. Giachetti, R. Maciocco, E. Sorace and M. Tarlini, Cohomological Properties of Differential Calculi on Hopf Algebras, Proocedings of the Symposium on Quantum Groups of the International Colloquium GROUP21, Goslar 1996, q-alg/9612019.

[47] L. Castellani, Gravity on finite groups, gr-qc/9909028.

[48] L. Castellani, Differential calculi on finite groups, JHEP, Proceedings of the Corfu Summer Inst. on Elementary Particle Phys., 1999, math.QA/0005226.

[49] A. Dimakis and F. Müller-Hoissen, Bidifferential calculi and integrable models, math-ph/9908015.

[50] A. Dimakis, F. Müller-Hoissen and T. Striker, Non-commutative differential calculus and lattice gauge theory, J.Phys. A26 (1993)1927.

[51] S.L. Woronowicz,Differential calculus on compact matrix pseudogroups (Quantum groups), Commun. Math. Phys. 122, (1989) 125.

[52] P. Aschieri, On the geometry of inhomogeneous quantum groups, Ph.D Thesis, Scuola Normale Superiore di Pisa (1998), math.QA/9805119.

[53] R. Coquereaux, G. Esposito-Farese and G. Vaillant, "Higgs fields as Yang-Mills fields and discrete symmetries," Nucl. Phys. B353, 689 (1991).

[54] J. Madore and J. Mourad, "Noncommutative Kaluza-Klein Theory," hepth/9601169.

[55] L. O'Raifeartaigh and N. Straumann, "Early history of gauge theories and Kaluza-Klein theories," hep-ph/9810524. 